\title{
(98)
}

\section{Balanza de Pagos}


7.1. BALANZA DE PAGOS: CUENTA CORRIENTE Y DE CAPITAL. SERIE HISTÓRICA*

(Millones de euros)

\begin{tabular}{|c|c|c|c|c|c|c|c|c|c|c|c|c|c|c|c|}
\hline \multirow[t]{2}{*}{ Año } & \multicolumn{3}{|c|}{$\begin{array}{l}\text { Balanza } \\
\text { comercial }\end{array}$} & \multicolumn{3}{|c|}{$\begin{array}{c}\text { Balanza } \\
\text { de servicios }{ }^{\star *}\end{array}$} & \multicolumn{3}{|c|}{$\begin{array}{c}\text { Balanza } \\
\text { de rentas }\end{array}$} & \multicolumn{3}{|c|}{$\begin{array}{c}\text { Bal. de transferencias } \\
\text { corrientes }+ \\
\text { balanza de capital }\end{array}$} & \multicolumn{3}{|c|}{$\begin{array}{c}\text { Balanza por } \\
\text { cuenta corriente + } \\
\text { balanza de capital }\end{array}$} \\
\hline & Ingr. & Pagos & Saldo & Ingr. & Pagos & Saldo & Ingr. & Pagos & Saldo & Ingr. & Pagos & Saldo & Ingr. & Pagos & Saldo \\
\hline 1961 & 274 & 374 & -100 & 192 & 70 & 122 & - & - & - & 61 & 2 & 58 & 527 & 447 & 80 \\
\hline 1962 & & & & & 91 & & - & - & - & 81 & 1 & & 630 & 612 & 18 \\
\hline 1963 & 284 & 48 & -365 & 324 & 119 & 205 & - & - & - & 96 & 2 & 93 & 703 & 770 & -67 \\
\hline 1964 & 362 & 48 & -386 & 423 & 141 & 282 & - & - & - & 117 & 1 & 115 & 902 & 890 & 11 \\
\hline 1965 & 367 & 1.002 & & 507 & 177 & 329 & - & - & - & 132 & 2 & 130 & 1.005 & & -175 \\
\hline 1966 & 472 & & -718 & 581 & 218 & 364 & - & - & - & 153 & 2 & 151 & 1.206 & 1.409 & -204 \\
\hline 1967 & 519 & & -652 & 570 & 250 & 320 & - & - & - & 167 & 2 & 165 & 1.256 & 1.423 & -167 \\
\hline 1968 & 701 & & -662 & 712 & 340 & & - & - & - & 195 & 7 & 188 & & & -102 \\
\hline 1969 & 839 & & -787 & 804 & 407 & & - & - & - & & 13 & & & & -166 \\
\hline 1970 & 1.045 & & & & 472 & & - & - & - & & & & & & 34 \\
\hline 1971 & 253 & & & & & & - & - & - & 340 & 17 & & & & 358 \\
\hline 1972 & 519 & 2 & -8 & & 6 & 7 & - & - & - & 368 & 33 & & & & 221 \\
\hline 1973 & 884 & & -1.236 & & 86 & 9 & - & - & - & 539 & 45 & 493 & & & 195 \\
\hline 1974 & 2.510 & 954 & -2.443 & 1.960 & 1.034 & & - & - & - & 468 & 72 & 396 & & & -1.121 \\
\hline 1975 & 694 & & -2.549 & & & & - & - & - & 498 & 103 & 394 & & & -1.204 \\
\hline 1976 & & & & & & & - & - & - & & & & & & 729 \\
\hline 1977 & & & & & & & - & - & - & & & & & & 885 \\
\hline 1978 & & & & & & & - & - & - & & & & 11. & & \\
\hline 979 & & & & & & & - & - & - & & & & & & 453 \\
\hline 1980 & 8.915 & 130 & -5.06 & & & & - & - & - & 867 & 19 & 671 & & & -1.534 \\
\hline 1981 & 11.487 & 17.1 & -5.68 & 6.548 & & & - & - & - & 1.019 & 22 & 790 & & & -1.421 \\
\hline 1982 & 13.357 & 19.8 & -6.510 & & & & - & - & - & & 25 & 915 & & & -1.423 \\
\hline 1983 & 17.225 & & -6.469 & 10.1 & & & - & - & - & & & & & & 9 \\
\hline 84 & & & & & & & - & - & - & & & & & & \\
\hline 885 & 24.961 & & -4.6 & & & & - & - & - & & & & & & \\
\hline 986 & 23.216 & & & & & & - & - & - & & & & & & 4.773 \\
\hline 987 & & & & & & & - & - & - & & & & & & 2.016 \\
\hline 1988 & 28.041 & & 124 & 32 & & & - & - & - & & & & & & -190 \\
\hline 1989 & 31.068 & 48.772 & 7.704 & 7 & & & & - & & & & & & & -6.068 \\
\hline 990 & 33.994 & & & & & & 4.756 & 6.914 & -2.158 & & & & & & -10.114 \\
\hline 1 & 37.4 & & & & & & & & & & & & & & \\
\hline & & & & & & & & & & & & & & & \\
\hline 93 & 47.341 & & & & & & & & & & & & & & \\
\hline 94 & 59.435 & 1.303 & & & & & & & & & & & & & -3.297 \\
\hline 1995 & 70.013 & & 3.948 & & & & & & & & & & & & 3.178 \\
\hline 1996 & 78.324 & 90.544 & 2.2 & & & & & & & & & 7.477 & & & 3.980 \\
\hline 1997 & 94.294 & & & & & & & & & & & & & & 5.204 \\
\hline 98 & & & & & & & & & & & & & & & -582 \\
\hline & & & & & & & & & & & & & & & -10.454 \\
\hline 000 & & & & & & & & & & & & & & & \\
\hline 001 & 1.239 & & & & & & & & -12.576 & & & & & & -21.433 \\
\hline 002 & 4.816 & & & & & & & & & & & & & & -16.102 \\
\hline 2003 & 9.754 & & -39.8 & & & & & & & & & & & & -19.311 \\
\hline 2004 & 48.967 & 2.627 & -53.660 & & & & & & & & & & & & -35.736 \\
\hline 2005 & 77.978 & & & & & & & & & & & 4.787 & & & \\
\hline 2006 & 75.808 & & & & & & & & & & & -309 & 332.973 & 415.091 & -82.118 \\
\hline 2007 & 90.498 & 83.739 & -93.241 & & & & & & & & & -2.570 & & 469.591 & -100.689 \\
\hline 2008 & 92.743 & 279.781 & -87.038 & & & & & & & & & -3.914 & 367.952 & 7.153 & -99.201 \\
\hline 2009 & 3.929 & 5.401 & 11 & 80.7 & & 29.5 & & & & & & -3.806 & & 9.121 & \\
\hline 2010 & 90.835 & 38.639 & & & & & & & & & & -608 & 348.790 & & -34.420 \\
\hline 2011 & 215.968 & & & & & & & & & & & & & & \\
\hline 2012 & 24.324 & 252.560 & & & & & & & & & & & & & 12.261 \\
\hline 2013 & 234.000 & 246.607 & -12.607 & 97.076 & 48.737 & 48.339 & 36.884 & 52.161 & -15.277 & 29.741 & 27.898 & 1.843 & 396.463 & 374.500 & 21.963 \\
\hline
\end{tabular}

*Se mantiene esta serie histórica hasta 2013 con los cambios metodológicos sucesivos aplicados hasta la incorporación del V Manual de Balanza de Pagos del FMI.

${ }^{* *}$ Hasta 1990 la balanza de servicios contiene conjuntamente servicios y rentas.

En el cuadro 7.3 se pueden consultar los datos con la aplicación del VI Manual de Balanza de Pagos del FMI.

Fuente: Banco de España. 


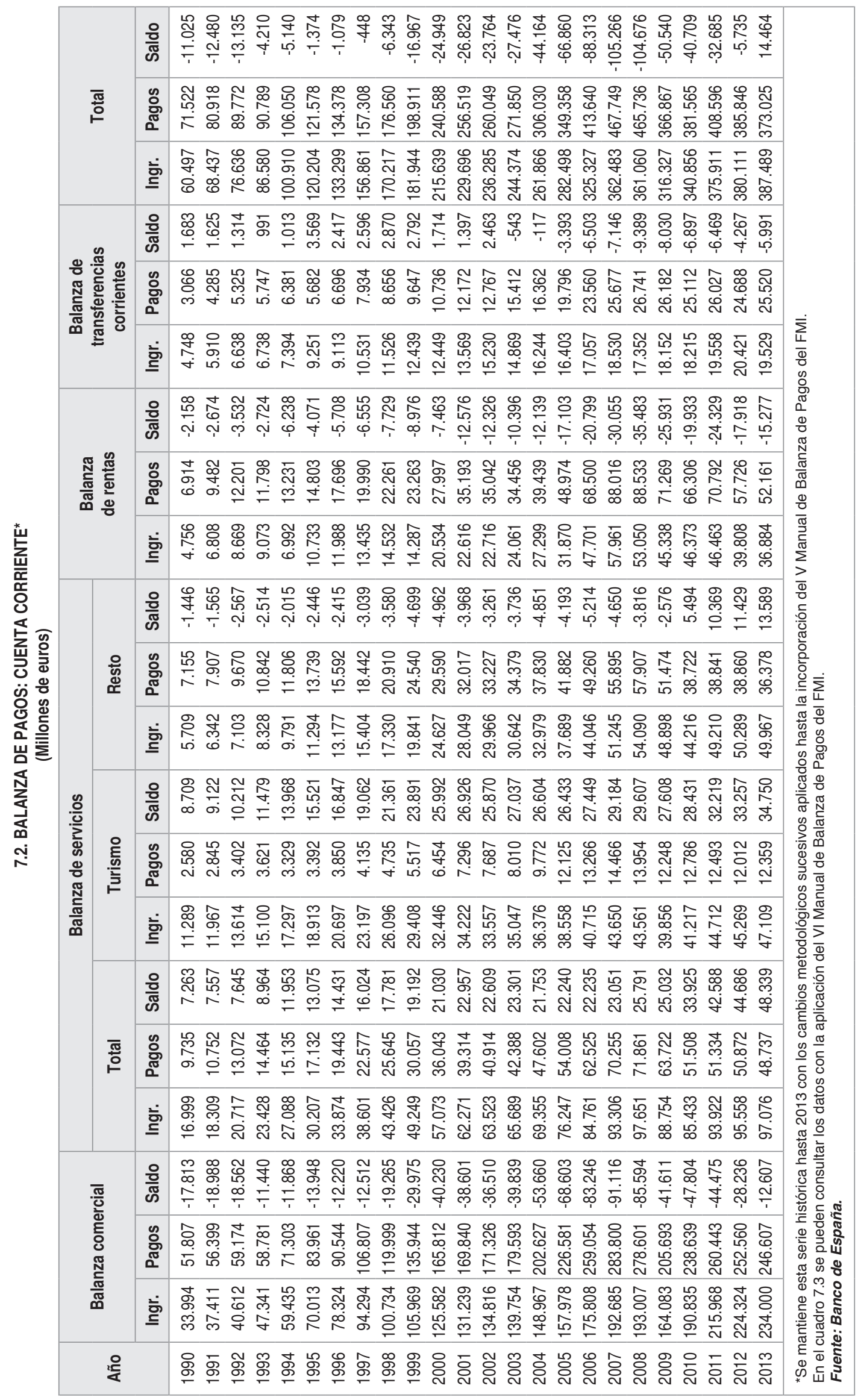




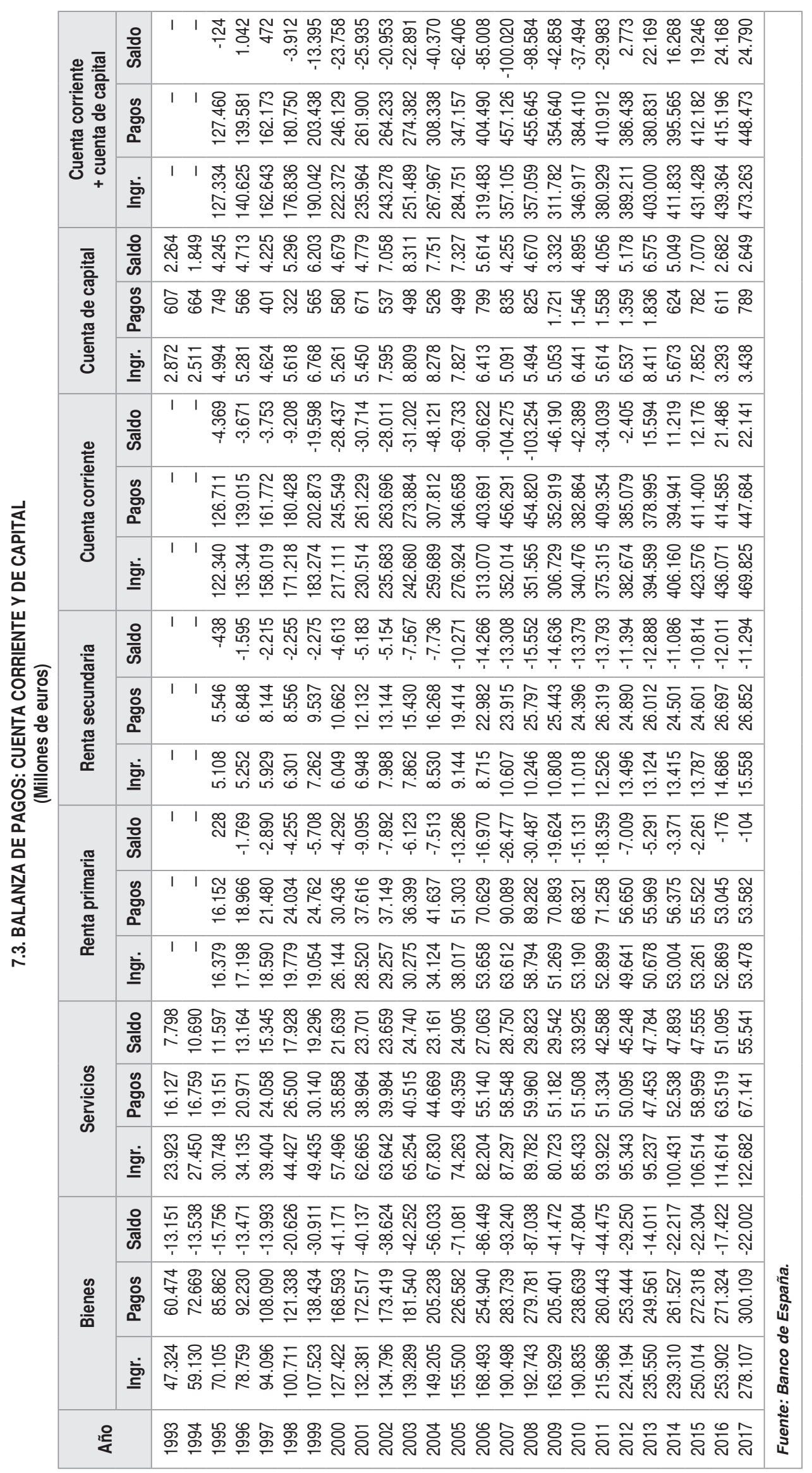


7.4. BALANZA DE PAGOS: CUENTA CORRIENTE Y DE CAPITAL (MBP5)* (Porcentaje del PIB)

\begin{tabular}{|c|c|c|c|c|c|c|c|c|c|c|c|c|c|c|c|}
\hline \multirow[t]{2}{*}{ Año } & \multicolumn{3}{|c|}{$\begin{array}{l}\text { Balanza } \\
\text { comercial }\end{array}$} & \multicolumn{3}{|c|}{$\begin{array}{c}\text { Balanza } \\
\text { de servicios }{ }^{* *}\end{array}$} & \multicolumn{3}{|c|}{$\begin{array}{c}\text { Balanza } \\
\text { de rentas }{ }^{\star *}\end{array}$} & \multicolumn{3}{|c|}{$\begin{array}{c}\text { Balanza de transferencias } \\
\text { corrientes y de capital }\end{array}$} & \multicolumn{3}{|c|}{$\begin{array}{l}\text { Balanza por } \\
\text { cuenta corriente } \\
\text { y de capital }\end{array}$} \\
\hline & Ingr. & Pagos & Saldo & Ingr. & Pagos & Saldo & Ingr. & Pagos & Saldo & Ingr. & Pagos & Saldo & Ingr. & Pagos & Saldo \\
\hline 1961 & 5,8 & 8,0 & $-2,1$ & 4,1 & 1,5 & 2,6 & - & - & - & 1,3 & 0,1 & 1,2 & 11,2 & 9,5 & 1,7 \\
\hline 1962 & 5,3 & 9,5 & $-4,2$ & 4,8 & 1,7 & 3,1 & - & - & - & 1,5 & 0,0 & 1,5 & 11,6 & 11,2 & 0,3 \\
\hline 1963 & 4,4 & 10,1 & $-5,7$ & 5,0 & 1,9 & 3,2 & - & - & - & 1,5 & 0,0 & 1,4 & 10,9 & 12,0 & $-1,0$ \\
\hline 1964 & 5,0 & 10,3 & $-5,3$ & 5,8 & 1,9 & 3,9 & - & - & - & 1,6 & 0,0 & 1,6 & 12,4 & 12,3 & 0,2 \\
\hline 1965 & 4,4 & 11,9 & $-7,5$ & 6,0 & 2,1 & 3,9 & - & - & - & 1,6 & 0,0 & 1,5 & 11,9 & 14,0 & $-2,1$ \\
\hline 1966 & 4,8 & 12,2 & $-7,3$ & 5,9 & 2,2 & 3,7 & - & - & - & 1,6 & 0,0 & 1,5 & 12,3 & 14,4 & $-2,1$ \\
\hline 1967 & 4,7 & 10,6 & $-5,9$ & 5,1 & 2,3 & 2,9 & - & - & - & 1,5 & 0,0 & 1,5 & 11,3 & 12,9 & $-1,5$ \\
\hline 1968 & 5,6 & 10,9 & $-5,3$ & 5,7 & 2,7 & 3,0 & - & - & - & 1,6 & 0,1 & 1,5 & 12,9 & 13,7 & $-0,8$ \\
\hline 1969 & 5,9 & 11,4 & $-5,5$ & 5,6 & 2,8 & 2,8 & - & - & - & 1,7 & 0,1 & 1,6 & 13,1 & 14,3 & $-1,2$ \\
\hline 1970 & 6,6 & 11,6 & $-5,0$ & 6,4 & 3,0 & 3,4 & - & - & - & 1,8 & 0,0 & 1,8 & 14,8 & 14,6 & 0,2 \\
\hline 1971 & 7,0 & 10,8 & $-3,8$ & 7,0 & 3,0 & 4,0 & - & - & - & 1,9 & 0,1 & 1,8 & 15,9 & 13,9 & 2,0 \\
\hline 1972 & 7,3 & 11,5 & $-4,3$ & 6,9 & 3,2 & 3,7 & - & - & - & 1,8 & 0,2 & 1,6 & 15,9 & 14,9 & 1,1 \\
\hline 1973 & 7,5 & 12,4 & $-4,9$ & 7,2 & 3,4 & 3,7 & - & - & - & 2,1 & 0,2 & 2,0 & 16,8 & 16,0 & 0,8 \\
\hline 1974 & 8,1 & 16,0 & $-7,9$ & 6,3 & 3,3 & 3,0 & - & - & - & 1,5 & 0,2 & 1,3 & 16,0 & 19,6 & $-3,6$ \\
\hline 1975 & 7,4 & 14,4 & $-7,0$ & 5,8 & 3,2 & 2,6 & - & - & - & 1,4 & 0,3 & 1,1 & 14,6 & 17,9 & $-3,3$ \\
\hline 1976 & 8,3 & 15,0 & $-6,7$ & 5,1 & 3,4 & 1,7 & - & - & - & 1,4 & 0,3 & 1,1 & 14,8 & 18,7 & $-4,0$ \\
\hline 1977 & 8,7 & 13,7 & $-5,1$ & 5,6 & 3,3 & 2,3 & - & - & - & 1,3 & 0,1 & 1,2 & 15,6 & 17,2 & $-1,6$ \\
\hline 1978 & 9,1 & 11,9 & $-2,7$ & 6,0 & 3,3 & 2,7 & - & - & - & 1,3 & 0,1 & 1,1 & 16,4 & 15,3 & 1,1 \\
\hline 1979 & 9,3 & 12,2 & $-2,9$ & 5,9 & 3,4 & 2,5 & - & - & - & 1,1 & 0,2 & 0,9 & 16,3 & 15,7 & 0,6 \\
\hline 1980 & 9,4 & 14,7 & $-5,3$ & 5,4 & 2,4 & 3,0 & - & - & - & 0,9 & 0,2 & 0,7 & 15,7 & 17,3 & $-1,6$ \\
\hline 1981 & 10,7 & 16,1 & $-5,3$ & 6,1 & 2,9 & 3,2 & - & - & - & 1,0 & 0,2 & 0,7 & 17,8 & 19,1 & $-1,3$ \\
\hline 1982 & 10,9 & 16,2 & $-5,3$ & 6,4 & 3,0 & 3,4 & - & - & - & 1,0 & 0,2 & 0,7 & 18,2 & 19,4 & $-1,2$ \\
\hline 1983 & 12,3 & 16,9 & $-4,6$ & 7,2 & 3,3 & 3,9 & - & - & - & 1,0 & 0,3 & 0,7 & 20,5 & 20,5 & 0,0 \\
\hline 1984 & 13,9 & 16,5 & $-2,6$ & 7,8 & 3,1 & 4,7 & - & - & - & 0,9 & 0,3 & 0,7 & 22,6 & 19,9 & 2,7 \\
\hline 1985 & 14,2 & 16,9 & $-2,7$ & 7,5 & 3,2 & 4,3 & - & - & - & 1,0 & 0,4 & 0,6 & 22,7 & 20,4 & 2,3 \\
\hline 1986 & 11,5 & 14,3 & $-2,7$ & 7,5 & 2,8 & 4,6 & - & - & - & 1,2 & 0,7 & 0,5 & 20,2 & 17,8 & 2,4 \\
\hline 1987 & 11,3 & 15,6 & $-4,2$ & 7,2 & 2,9 & 4,3 & - & - & - & 1,5 & 0,7 & 0,9 & 20,0 & 19,1 & 0,9 \\
\hline 1988 & 11,2 & 16,2 & $-5,0$ & 7,0 & 3,3 & 3,7 & - & - & - & 2,1 & 0,8 & 1,2 & 20,2 & 20,3 & $-0,1$ \\
\hline 1989 & 11,1 & 17,4 & $-6,3$ & 6,4 & 3,4 & 3,0 & - & - & - & 2,2 & 1,0 & 1,2 & 19,6 & 21,8 & $-2,2$ \\
\hline 1990 & 10,9 & 16,6 & $-5,7$ & 5,4 & 3,1 & 2,3 & 1,5 & 2,2 & $-0,7$ & 1,9 & 1,0 & 0,8 & 19,7 & 23,0 & $-3,2$ \\
\hline 1991 & 10,9 & 16,5 & $-5,5$ & 5,3 & 3,1 & 2,2 & 2,0 & 2,8 & $-0,8$ & 2,4 & 1,3 & 1,1 & 20,7 & 23,7 & $-3,0$ \\
\hline 1992 & 11,0 & 16,0 & $-5,0$ & 5,6 & 3,5 & 2,1 & 2,3 & 3,3 & $-1,0$ & 2,5 & 1,5 & 1,0 & 21,5 & 24,4 & $-2,9$ \\
\hline 1993 & 12,4 & 15,4 & $-3,0$ & 6,1 & 3,8 & 2,3 & 2,4 & 3,1 & $-0,7$ & 2,6 & 1,7 & 0,9 & 23,5 & 24,0 & $-0,5$ \\
\hline 1994 & 14,6 & 17,6 & $-2,9$ & 6,7 & 3,7 & 2,9 & 1,7 & 3,3 & $-1,5$ & 2,5 & 1,8 & 0,7 & 25,6 & 26,4 & $-0,8$ \\
\hline 1995 & 15,2 & 18,3 & $-3,0$ & 6,6 & 3,7 & 2,8 & 2,3 & 3,2 & $-0,9$ & 3,2 & 1,4 & 1,8 & 27,4 & 26,7 & 0,7 \\
\hline 1996 & 16,1 & 18,6 & $-2,5$ & 6,9 & 4,0 & 3,0 & 2,5 & 3,6 & $-1,2$ & 3,1 & 1,5 & 1,5 & 28,5 & 27,7 & 0,8 \\
\hline 1997 & 18,2 & 20,6 & $-2,4$ & 7,5 & 4,4 & 3,1 & 2,6 & 3,9 & $-1,3$ & 3,3 & 1,7 & 1,6 & 31,5 & 30,5 & 1,0 \\
\hline 1998 & 18,2 & 21,7 & $-3,5$ & 7,8 & 4,6 & 3,2 & 2,6 & 4,0 & $-1,4$ & 3,2 & 1,7 & 1,6 & 31,9 & 32,0 & $-0,1$ \\
\hline 1999 & 17,8 & 22,9 & $-5,0$ & 8,3 & 5,1 & 3,2 & 2,4 & 3,9 & $-1,5$ & 3,4 & 1,8 & 1,6 & 31,9 & 33,6 & $-1,8$ \\
\hline 2000 & 19,4 & 25,7 & $-6,2$ & 8,8 & 5,6 & 3,3 & 3,2 & 4,3 & $-1,2$ & 2,9 & 1,8 & 1,1 & 34,3 & 37,4 & $-3,1$ \\
\hline 2001 & 18,8 & 24,3 & $-5,5$ & 8,9 & 5,6 & 3,3 & 3,2 & 5,0 & $-1,8$ & 2,9 & 1,9 & 1,0 & 33,8 & 36,8 & $-3,1$ \\
\hline 2002 & 18,0 & 22,9 & $-4,9$ & 8,5 & 5,5 & 3,0 & 3,0 & 4,7 & $-1,6$ & 3,2 & 1,8 & 1,4 & 32,7 & 34,8 & $-2,1$ \\
\hline 2003 & 17,4 & 22,4 & $-5,0$ & 8,2 & 5,3 & 2,9 & 3,0 & 4,3 & $-1,3$ & 3,1 & 2,1 & 0,9 & 31,6 & 34,0 & $-2,4$ \\
\hline 2004 & 17,3 & 23,5 & $-6,2$ & 8,1 & 5,5 & 2,5 & 3,2 & 4,6 & $-1,4$ & 3,0 & 2,0 & 1,0 & 31,5 & 35,6 & $-4,1$ \\
\hline 2005 & 17,0 & 24,3 & $-7,4$ & 8,2 & 5,8 & 2,4 & 3,4 & 5,3 & $-1,8$ & 2,7 & 2,2 & 0,5 & 31,3 & 37,6 & $-6,3$ \\
\hline 2006 & 17,4 & 25,7 & $-8,3$ & 8,4 & 6,2 & 2,2 & 4,7 & 6,8 & $-2,1$ & 2,5 & 2,5 & 0,0 & 33,0 & 41,2 & $-8,1$ \\
\hline 2007 & 17,6 & 26,3 & $-8,6$ & 8,1 & 5,4 & 2,7 & 5,4 & 8,1 & $-2,8$ & 2,3 & 2,5 & $-0,2$ & 34,1 & 43,4 & $-9,3$ \\
\hline 2008 & 17,3 & 25,1 & $-7,8$ & 8,0 & 5,4 & 2,7 & 4,8 & 7,9 & $-3,2$ & 2,2 & 2,5 & $-0,4$ & 33,0 & 41,9 & $-8,9$ \\
\hline 2009 & 15,2 & 19,0 & $-3,8$ & 7,5 & 4,7 & 2,7 & 4,2 & 6,6 & $-2,4$ & 2,3 & 2,6 & $-0,4$ & 29,9 & 34,2 & $-4,3$ \\
\hline 2010 & 17,7 & 22,1 & $-4,4$ & 7,9 & 4,8 & 3,1 & 4,3 & 6,1 & $-1,8$ & 2,4 & 2,5 & $-0,1$ & 32,3 & 35,5 & $-3,2$ \\
\hline 2011 & 20,2 & 24,3 & $-4,2$ & 8,8 & 4,8 & 4,0 & 4,3 & 6,6 & $-2,3$ & 2,5 & 2,6 & $-0,1$ & 35,6 & 38,4 & $-2,8$ \\
\hline 2012 & 21,6 & 24,3 & $-2,7$ & 9,2 & 4,9 & 4,3 & 3,8 & 5,6 & $-1,7$ & 2,7 & 2,5 & 0,2 & 38,2 & 37,1 & 1,2 \\
\hline 2013 & 22,8 & 24,0 & $-1,2$ & 9,5 & 4,8 & 4,7 & 3,6 & 5,1 & $-1,5$ & 2,9 & 2,7 & 0,2 & 38,7 & 36,5 & 2,1 \\
\hline
\end{tabular}

*Se mantiene esta serie histórica hasta 2013 con los cambios metodológicos sucesivos aplicados hasta la incorporación del V Manual de Balanza de Pagos del FMI.

${ }^{* *}$ Hasta 1990 la balanza de servicios contiene conjuntamente servicios y rentas.

En el cuadro 7.5 se pueden consultar los datos con la aplicación del VI Manual de Balanza de Pagos del FMI.

Fuente: elaboración propia a partir de datos del Banco de España e INE. PIB, Contabilidad Nacional, base 1986 hasta 1979 , base 1995 hasta 1994 y base 2010 en adelante. 
7.5. BALANZA DE PAGOS: CUENTA CORRIENTE Y DE CAPITAL (MBP6)

(Porcentaje del PIB)

\begin{tabular}{|c|c|c|c|c|c|c|c|c|c|c|c|c|c|c|c|c|c|c|c|c|c|}
\hline \multirow[t]{2}{*}{ Año } & \multicolumn{3}{|c|}{ Bienes } & \multicolumn{3}{|c|}{ Servicios } & \multicolumn{3}{|c|}{ Renta primaria } & \multicolumn{3}{|c|}{$\begin{array}{c}\text { Renta } \\
\text { secundaria }\end{array}$} & \multicolumn{3}{|c|}{$\begin{array}{l}\text { Cuenta } \\
\text { corriente }\end{array}$} & \multicolumn{3}{|c|}{$\begin{array}{l}\text { Cuenta } \\
\text { de capital }\end{array}$} & \multicolumn{3}{|c|}{$\begin{array}{l}\text { Cuenta corriente } \\
+ \text { + cuenta de capital }\end{array}$} \\
\hline & Ingr. & Pagos & Saldo & Ingr. & Pagos & Saldo & Ingr. & Pagos & Saldo & Ingr. & Pagos & Saldo & Ingr. & Pagos & Saldo & Ingr. & Pagos & Saldo & Ingr. & Pagos & Saldo \\
\hline 1993 & 12,4 & 5,8 & $-3,4$ & 6,3 & 4,2 & 2,0 & - & - & - & - & - & - & - & - & - & 0,8 & 0,2 & 0,6 & - & - & - \\
\hline 1994 & 4,6 & 17,9 & $-3,3$ & 6,8 & 4,1 & 2,6 & - & - & - & - & - & - & - & - & - & 0,6 & 0,2 & 0,5 & - & - & - \\
\hline 1995 & 5,3 & 18,7 & $-3,4$ & 6,7 & 4,2 & 2,5 & 3,6 & 3,5 & 0,0 & 1,1 & 1,2 & $-0,1$ & 26,6 & 27,6 & $-1,0$ & 1,1 & 0,2 & 0,9 & 27,7 & 27,7 & 0,0 \\
\hline 1996 & 16,1 & 18,9 & $-2,8$ & 7,0 & 4,3 & 2,7 & 3,5 & 3,9 & $-0,4$ & 1,1 & 1,4 & $-0,3$ & 27,7 & 28,5 & $-0,8$ & 1,1 & 0,1 & 0 & 28,8 & 28,6 & 0,2 \\
\hline 1997 & 18,2 & 20,9 & $-2,7$ & 7,6 & 4,6 & 3,0 & 3,6 & 4,1 & $-0,6$ & 1,1 & 1,6 & $-0,4$ & 30,5 & 31,2 & $-0,7$ & 0,9 & 0,1 & 0,8 & 31,4 & 31,3 & 0,1 \\
\hline 1998 & 18,2 & 21,9 & $-3,7$ & 8,0 & 4,8 & 3,2 & 3,6 & 4,3 & $-0,8$ & 1,1 & & $-0,4$ & 30,9 & 32,6 & $-1,7$ & 1,0 & & 0 & 1,9 & 2,6 & $-0,7$ \\
\hline 1999 & 18,1 & 23,3 & $-5,2$ & 8,3 & 5,1 & 3,2 & 3,2 & 4,2 & $-1,0$ & 1,2 & 1,6 & $-0,4$ & 30,8 & 34,1 & $-3,3$ & 1,1 & 0,1 & 0 & 32,0 & 34,2 & $-2,3$ \\
\hline 2000 & 19,7 & 26,1 & $-6,4$ & 8,9 & 5,5 & 3,2 & 4,0 & 4,7 & $-0,7$ & 0,9 & 1,6 & $-0,7$ & 33,6 & 38,0 & $-4,4$ & 0,8 & 0,1 & 0,7 & 34,4 & 38,1 & $-3,7$ \\
\hline 2001 & 18,9 & 24,7 & $-5,7$ & 9,0 & 5,6 & 3,4 & 4,1 & 5,4 & $-1,3$ & 1,0 & 1,7 & $-0,7$ & 33,0 & 37,3 & $-4,4$ & 0,8 & 0,1 & 0,7 & 33,7 & 37,4 & $-3,7$ \\
\hline 2002 & 18,0 & 23,1 & $-5,2$ & 8 & 5,3 & 3,2 & 3,9 & 5,0 & $-1,1$ & 1,1 & 1 & $-0,7$ & 31,5 & 35,2 & $-3,7$ & 1,0 & 0,1 & 0,9 & 32,5 & 35,3 & $-2,8$ \\
\hline 2003 & 17,3 & 22,6 & $-5,3$ & 8, & 5, & 3 , & 3,8 & 4,5 & $-0,8$ & 1,0 & & $-0,9$ & 30,2 & 34,1 & $-3,9$ & 1,1 & & 0 & 31,3 & 34,1 & $-2,8$ \\
\hline 2004 & 17,3 & 23,8 & $-6,5$ & 7,9 & 5,2 & 2,7 & 4,0 & 4,8 & $-0,9$ & 1,0 & 1,9 & $-0,9$ & 30,1 & 35,7 & $-5,6$ & 1,0 & 0,1 & 0,9 & 1,1 & 35,8 & $-4,7$ \\
\hline 2005 & 6,7 & 24,3 & $-7,6$ & 8,0 & 5,3 & 2,7 & 4,1 & 5,5 & $-1,4$ & 1,0 & 2, & $-1,1$ & 29,8 & 37,3 & $-7,5$ & 0,8 & 0,1 & 0,8 & 30,6 & 37,3 & $-6,7$ \\
\hline 2006 & 16,7 & 25,3 & $-8,6$ & 8,2 & 5,5 & 2,7 & 5,3 & 7,0 & $-1,7$ & 0,9 & 2,3 & $-1,4$ & 31,1 & 40,0 & $-9,0$ & 0,6 & 0,1 & 0,6 & 31,7 & 40,1 & $-8,4$ \\
\hline 2007 & 17,6 & 26,3 & $-8,6$ & 8,1 & 5,4 & 2,7 & 5,9 & 8,3 & $-2,4$ & 1,0 & 2,2 & $-1,2$ & 32,6 & 42,2 & $-9,6$ & 0,5 & 0,1 & 0,4 & 33,0 & 42,3 & $-9,3$ \\
\hline 2008 & 17,3 & 25,1 & $-7,8$ & 8,0 & 5,4 & 2,7 & 5,3 & 8,0 & $-2,7$ & 0,9 & 2,3 & $-1,4$ & 31,5 & 40,7 & $-9,3$ & 0,5 & 0,1 & 0,4 & 32,0 & 40,8 & $-8,8$ \\
\hline 2009 & 15,2 & 19,0 & $-3,8$ & 7,5 & 4,7 & 2,7 & 4,8 & 6,6 & $-1,8$ & 1,0 & 2,4 & $-1,4$ & 28,4 & 32,7 & $-4,3$ & 0,5 & 0,2 & 0,3 & 28,9 & 32,9 & $-4,0$ \\
\hline 2010 & 17,7 & 22,1 & $-4,4$ & 7,9 & 4,8 & 3,1 & 4,9 & 6,3 & $-1,4$ & 1,0 & 2,3 & $-1,2$ & 31,5 & 35,4 & $-3,9$ & 0,6 & 0,1 & 0,5 & 32,1 & 35,6 & $-3,5$ \\
\hline 2011 & 20,2 & 24,3 & $-4,2$ & 8,8 & 4,8 & 4,0 & 4,9 & 6,7 & -1 & 1,2 & 2 , & $-1,3$ & 35,1 & 38,2 & $-3,2$ & 0,5 & 0,1 &, 4 & 35,6 & 38,4 & $-2,8$ \\
\hline 2012 & 21,6 & 24,4 & $-2,8$ & 9,2 & 4,8 & 4,4 & 4,8 & 5,4 & $-0,7$ & 1,3 & 2,4 & $-1,1$ & 36,8 & 37,0 & $-0,2$ & 0,6 & 0,1 & 0,5 & 37,4 & 37,2 & 0,3 \\
\hline 2013 & 23,0 & 24,3 & $-1,4$ & 9,3 & 4,6 & 4,7 & 4,9 & 5,5 & $-0,5$ & 1,3 & 2,5 & $-1,3$ & 38,5 & 37,0 & 1,5 & 0,8 & 0,2 & 0,6 & 39,3 & 37,1 & 2,2 \\
\hline 2014 & 23,1 & 25,2 & $-2,1$ & 9,7 & 5,1 & 4,6 & 5,1 & 5,4 & $-0,3$ & 1,3 & 2,4 & $-1,1$ & 39,1 & 38,1 & 1,1 & 0,5 & 0,1 & 0,5 & 39,7 & 38,1 & 1,6 \\
\hline 2015 & 23,1 & 25,2 & $-2,1$ & 9,9 & 5,5 & 4,4 & 4,9 & 5,1 & $-0,2$ & 1,3 & 2,3 & $-1,0$ & 39,2 & 38,1 & 1,1 & 0,7 & 0,1 & 0,7 & 39,9 & 38,2 & 1,8 \\
\hline 2016 & 22,7 & 24,3 & $-1,6$ & 10,2 & 5,7 & 4,6 & 4,7 & 4,7 & 0,0 & 1,3 & 2,4 & $-1,1$ & 39,0 & 37,1 & 1,9 & 0,3 & 0,1 & 0,2 & 39,3 & 37,1 & 2,2 \\
\hline 2017 & 23,9 & 25,8 & $-1,9$ & 10,5 & 5,8 & 4,8 & 4,6 & 4,6 & 0,0 & 1,3 & 2,3 & $-1,0$ & 40,4 & 38,5 & 1,9 & 0,3 & 0,1 & 0,2 & 40,7 & 38,5 & 2,1 \\
\hline
\end{tabular}

Fuente: elaboración propia a partir de datos del Banco de España e INE. PIB, Contabilidad Nacional, base 1995 hasta 1994 y base 2010 en adelante. 
7.6. BALANZA DE PAGOS: CUENTA FINANCIERA*

(Millones de euros)

\begin{tabular}{|c|c|c|c|c|c|c|c|c|c|c|c|}
\hline \multirow{3}{*}{ Año } & \multicolumn{7}{|c|}{ Excluido el Banco de España } & \multicolumn{4}{|c|}{ Banco de España } \\
\hline & \multicolumn{3}{|c|}{ Inversión extranjera en España } & \multicolumn{3}{|c|}{ Inversión española en el exterior } & \multirow[b]{2}{*}{$\begin{array}{l}\text { Derivados } \\
\text { VNA-VNP }\end{array}$} & \multirow[b]{2}{*}{$\begin{array}{c}\text { Total } \\
\text { VNA-VNP } \\
(4)\end{array}$} & \multirow[b]{2}{*}{ Reservas } & \multirow{2}{*}{$\begin{array}{c}\text { Posición } \\
\text { neta BE } \\
\text { frente } \\
\text { al Euro- } \\
\text { sistema }\end{array}$} & \multirow[b]{2}{*}{$\begin{array}{l}\text { Otros } \\
\text { BE }\end{array}$} \\
\hline & $\begin{array}{l}\text { Inversión } \\
\text { directa } \\
\text { (1) }\end{array}$ & $\begin{array}{l}\text { Inversión } \\
\text { en cartera } \\
(2)\end{array}$ & $\begin{array}{c}\text { Otra } \\
\text { inversión } \\
\text { (3) }\end{array}$ & $\begin{array}{l}\text { Inversión } \\
\text { directa }\end{array}$ & $\begin{array}{l}\text { Inversión } \\
\text { en cartera }\end{array}$ & $\begin{array}{c}\text { Otra } \\
\text { inversión }\end{array}$ & & & & & \\
\hline 1990 & 8.479 & 6.152 & 10.174 & 2.109 & 822 & 8.023 & 7 & 4.266 & 4.266 & - & - \\
\hline 1991 & 7.774 & 13.698 & 7.125 & 2.764 & 1.455 & 4.369 & -51 & 8.950 & 8.950 & - & - \\
\hline 1992 & 8.216 & 7.502 & 15.745 & 1.336 & 1.688 & 24.716 & 81 & -10.686 & -10.686 & - & - \\
\hline 1993 & 8.224 & 41.625 & 14.384 & 3.330 & 5.078 & 53.993 & -2.137 & -3.642 & -3.734 & - & 92 \\
\hline 1994 & 7.092 & -16.996 & 9.538 & 2.934 & 1.239 & -7.977 & 115 & -65 & -43 & - & -22 \\
\hline 1995 & 6.195 & 15.875 & 4.720 & 3.643 & 328 & 27.765 & -444 & -5.046 & -5.085 & - & 39 \\
\hline 1996 & 7.470 & 2.356 & 13.257 & 5.518 & 2.806 & -1.230 & -669 & 18.106 & 18.462 & - & -356 \\
\hline 1997 & 8.302 & 11.066 & 18.220 & 13.136 & 14.402 & 1.774 & -5 & 10.738 & 10.352 & - & 386 \\
\hline 1998 & 13.988 & 15.400 & 41.061 & 19.379 & 39.069 & 20.489 & -2.457 & -11.867 & -12.248 & - & 381 \\
\hline 1999 & 18.647 & 42.095 & 38.140 & 42.716 & 44.090 & -14.041 & 274 & 15.068 & -20.970 & 31.581 & 4.457 \\
\hline 2000 & 44.754 & 63.644 & 56.862 & 64.989 & 65.029 & 11.541 & 1.916 & 6.144 & -3.302 & 9.250 & 195 \\
\hline 2001 & 32.587 & 31.303 & 32.544 & 37.836 & 50.228 & 3.742 & -400 & -17.475 & -1.581 & -16.122 & 228 \\
\hline 2002 & 41.167 & 35.900 & 36.500 & 34.258 & 31.264 & 31.407 & -4.916 & -3.561 & 3.630 & -6.506 & -685 \\
\hline 2003 & 27.397 & 39.042 & 64.721 & 29.968 & 65.767 & 15.876 & -3.435 & -1.575 & -13.626 & -4.382 & 16.433 \\
\hline 2004 & 20.234 & 112.754 & 20.207 & 49.187 & 27.123 & 28.419 & 74 & 14.010 & -5.147 & 13.760 & 5.397 \\
\hline 2005 & 21.875 & 138.474 & 64.603 & 35.387 & 80.043 & 47.253 & 366 & 2.114 & -1.439 & -14.855 & 18.409 \\
\hline 2006 & 25.991 & 195.685 & 34.445 & 84.540 & -3.374 & 66.093 & 2.003 & 25.800 & 480 & 12.327 & 12.993 \\
\hline 2007 & 52.347 & 95.517 & 95.831 & 105.541 & -8.036 & 56.132 & -4.094 & -14.322 & 164 & -28.329 & 13.843 \\
\hline 2008 & 53.651 & -21.963 & 88.048 & 52.122 & -21.003 & 12.332 & -7.066 & -30.218 & 645 & -31.713 & 850 \\
\hline 2009 & 9.812 & 48.316 & 8.673 & 11.756 & 4.281 & 4.009 & -6.052 & -10.462 & 4.327 & -6.144 & -8.645 \\
\hline 2010 & 30.449 & -35.967 & 6.395 & 28.987 & -64.366 & 17.627 & 8.607 & -15.694 & 816 & -9.788 & -6.722 \\
\hline 2011 & 23.294 & -68.194 & -8.066 & 32.520 & -41.942 & 33.895 & -2.068 & -109.231 & 10.022 & -124.056 & 4.803 \\
\hline 2012 & 19.189 & -51.644 & -104.556 & -1.929 & 3.758 & 40.016 & 8.348 & -168.759 & 2.211 & -154.604 & -16.366 \\
\hline 2013 & 39.292 & 48.572 & -24.631 & 20.756 & -4.419 & -39.033 & -1.040 & 118.185 & 535 & 136.688 & -19.038 \\
\hline 2014 & 25.121 & 57.263 & 6.196 & 31.601 & 51.818 & -7.869 & -1.283 & 27.495 & 3.872 & 46.972 & -23.349 \\
\hline 2015 & 30.911 & 74.429 & -35.946 & 58.837 & 67.633 & 7.790 & -1.012 & -40.163 & 5.067 & -50.929 & 5.699 \\
\hline 2016 & 28.699 & -12.733 & -19.763 & 45.371 & 25.559 & 7.222 & -2.622 & -52.633 & 8.233 & -59.707 & -1.159 \\
\hline 2017 & 4.955 & 64.555 & 2.375 & 24.210 & 80.510 & 23.616 & -2.056 & -31.945 & 3.652 & -31.266 & -4.331 \\
\hline
\end{tabular}

VNA: Variación Neta de Activos. VNP: Variación Neta de Pasivos.

(1) No recoge las inversiones directas en acciones cotizadas; incluye las inversiones de cartera en acciones no cotizadas.

(2) Incluye las inversiones directas en acciones cotizadas; no recoge las inversiones en cartera en acciones no cotizadas.

(3) Incluye, principalmente, préstamos, repos y depósitos.

(4) Incluye todos los saldos exteriores del Banco de España (reservas, activos frente al Eurosistema y otros activos netos).

*VI Manual Balanza de Pagos a partir del año 2012.

Fuente: elaboración propia a partir de datos del Banco de España e INE. 
7.7. BALANZA DE PAGOS: CUENTA FINANCIERA*

(Porcentaje del PIB)

\begin{tabular}{|c|c|c|c|c|c|c|c|c|c|c|c|}
\hline \multirow{3}{*}{ Año } & \multicolumn{7}{|c|}{ Excluido el Banco de España } & \multicolumn{4}{|c|}{ Banco de España } \\
\hline & \multicolumn{3}{|c|}{ Inversión extranjera en España } & \multicolumn{3}{|c|}{ Inversión española en el exterior } & \multirow[b]{2}{*}{$\begin{array}{l}\text { Derivados } \\
\text { VNA-VNP }\end{array}$} & \multirow{2}{*}{$\begin{array}{c}\text { Total } \\
\text { VNA-VNP } \\
(4)\end{array}$} & \multirow[b]{2}{*}{ Reservas } & \multirow{2}{*}{$\begin{array}{l}\text { Activos } \\
\text { netos } \\
\text { frente } \\
\text { al Euro- } \\
\text { sistema }\end{array}$} & \multirow{2}{*}{$\begin{array}{c}\text { Otros } \\
\text { activos } \\
\text { netos }\end{array}$} \\
\hline & $\begin{array}{l}\text { Inversión } \\
\text { directa } \\
\text { (1) }\end{array}$ & $\begin{array}{c}\text { Inversión } \\
\text { en cartera } \\
(2)\end{array}$ & $\begin{array}{c}\text { Otra } \\
\text { inversión } \\
(3)\end{array}$ & $\begin{array}{l}\text { Inversión } \\
\text { directa }\end{array}$ & $\begin{array}{l}\text { Inversión } \\
\text { en cartera }\end{array}$ & $\begin{array}{c}\text { Otra } \\
\text { inversión }\end{array}$ & & & & & \\
\hline 1990 & 2,7 & 2,0 & 3,3 & 0,7 & 0,3 & 2,6 & - & 1,4 & 1,4 & - & - \\
\hline 1991 & 2,3 & 4,0 & 2,1 & 0,8 & 0,4 & 1,3 & 0,0 & 2,6 & 2,6 & - & - \\
\hline 1992 & 2,2 & 2,0 & 4,3 & 0,4 & 0,5 & 6,7 & 0,0 & $-2,9$ & $-2,9$ & - & - \\
\hline 1993 & 2,2 & 10,9 & 3,8 & 0,9 & 1,3 & 14,1 & $-0,6$ & $-1,0$ & $-1,0$ & - & 0,0 \\
\hline 1994 & 1,7 & $-4,2$ & 2,3 & 0,7 & 0,3 & $-2,0$ & 0,0 & 0,0 & 0,0 & - & 0,0 \\
\hline 1995 & 1,3 & 3,5 & 1,0 & 0,8 & 0,1 & 6,0 & $-0,1$ & $-1,1$ & $-1,1$ & - & 0,0 \\
\hline 1996 & 1,5 & 0,5 & 2,7 & 1,1 & 0,6 & $-0,3$ & $-0,1$ & 3,7 & 3,8 & - & $-0,1$ \\
\hline 1997 & 1,6 & 2,1 & 3,5 & 2,5 & 2,8 & 0,3 & 0,0 & 2,1 & 2,0 & - & 0,1 \\
\hline 1998 & 2,5 & 2,8 & 7,4 & 3,5 & 7,1 & 3,7 & $-0,4$ & $-2,1$ & $-2,2$ & - & 0,1 \\
\hline 1999 & 3,1 & 7,1 & 6,4 & 7,2 & 7,4 & $-2,4$ & 0,0 & 2,5 & $-3,5$ & 5,3 & 0,7 \\
\hline 2000 & 6,9 & 9,8 & 8,8 & 10,1 & 10,1 & 1,8 & 0,3 & 1,0 & $-0,5$ & 1,4 & 0,0 \\
\hline 2001 & 4,7 & 4,5 & 4,7 & 5,4 & 7,2 & 0,5 & $-0,1$ & $-2,5$ & $-0,2$ & $-2,3$ & 0,0 \\
\hline 2002 & 5,5 & 4,8 & 4,9 & 4,6 & 4,2 & 4,2 & $-0,7$ & $-0,5$ & 0,5 & $-0,9$ & $-0,1$ \\
\hline 2003 & 3,4 & 4,9 & 8,1 & 3,7 & 8,2 & 2,0 & $-0,4$ & $-0,2$ & $-1,7$ & $-0,5$ & 2,0 \\
\hline 2004 & 2,3 & 13,1 & 2,3 & 5,7 & 3,1 & 3,3 & 0,0 & 1,6 & $-0,6$ & 1,6 & 0,6 \\
\hline 2005 & 2,4 & 14,9 & 6,9 & 3,8 & 8,6 & 5,1 & 0,0 & 0,2 & $-0,2$ & $-1,6$ & 2,0 \\
\hline 2006 & 2,6 & 19,4 & 3,4 & 8,4 & $-0,3$ & 6,6 & 0,2 & 2,6 & 0,0 & 1,2 & 1,3 \\
\hline 2007 & 4,8 & 8,8 & 8,9 & 9,8 & $-0,7$ & 5,2 & $-0,4$ & $-1,3$ & 0,0 & $-2,6$ & 1,3 \\
\hline 2008 & 4,8 & $-2,0$ & 7,9 & 4,7 & $-1,9$ & 1,1 & $-0,6$ & $-2,7$ & 0,1 & $-2,8$ & 0,1 \\
\hline 2009 & 0,9 & 4,5 & 0,8 & 1,1 & 0,4 & 0,4 & $-0,6$ & $-1,0$ & 0,4 & $-0,6$ & $-0,8$ \\
\hline 2010 & 2,8 & $-3,3$ & 0,6 & 2,7 & $-6,0$ & 1,6 & 0,8 & $-1,5$ & 0,1 & $-0,9$ & $-0,6$ \\
\hline 2011 & 2,2 & $-6,4$ & $-0,8$ & 3,0 & $-3,9$ & 3,2 & $-0,2$ & $-10,2$ & 0,9 & $-11,6$ & 0,4 \\
\hline 2012 & 1,8 & $-5,0$ & $-10,1$ & $-0,2$ & 0,4 & 3,8 & 0,8 & $-16,2$ & 0,2 & $-14,9$ & $-1,6$ \\
\hline 2013 & 3,8 & 4,7 & $-2,4$ & 2,0 & $-0,4$ & $-3,8$ & $-0,1$ & 11,5 & 0,1 & 13,3 & $-1,9$ \\
\hline 2014 & 2,4 & 5,5 & 0,6 & 3,0 & 5,0 & $-0,8$ & $-0,1$ & 2,6 & 0,4 & 4,5 & $-2,2$ \\
\hline 2015 & 2,9 & 6,9 & $-3,3$ & 5,4 & 6,3 & 0,7 & $-0,1$ & $-3,7$ & 0,5 & $-4,7$ & 0,5 \\
\hline 2016 & 2,6 & $-1,1$ & $-1,8$ & 4,1 & 2,3 & 0,6 & $-0,2$ & $-4,7$ & 0,7 & $-5,3$ & $-0,1$ \\
\hline 2017 & 0,4 & 5,5 & 0,2 & 2,1 & 6,9 & 2,0 & $-0,2$ & $-2,7$ & 0,3 & $-2,7$ & $-0,4$ \\
\hline
\end{tabular}

VNA: Variación Neta de Activos. VNP: Variación Neta de Pasivos.

(1) No recoge las inversiones directas en acciones cotizadas; incluye las inversiones de cartera en acciones no cotizadas.

(2) Incluye las inversiones directas en acciones cotizadas; no recoge las inversiones en cartera en acciones no cotizadas.

(3) Incluye, principalmente, préstamos, repos y depósitos.

(4) Incluye todos los saldos exteriores del Banco de España (reservas, activos frente al Eurosistema y otros activos netos).

*VI Manual Balanza de Pagos a partir del año 2012.

Fuente: elaboración propia a partir de datos del Banco de España e INE. PIB, Contabilidad Nacional, base 1995 hasta 1994 y base 2010 en adelante. 